\title{
Metabolic and Structural Skeletal Muscle Health in Systemic Lupus Erythematosus-Related Fatigue: A Multimodal Magnetic Resonance Imaging Study
}

\author{
Sai Man Cheung, ${ }^{1}$ Karen Keenan, ${ }^{1}$ Nicholas Senn, ${ }^{1}$ Gayle Hutcheon, ${ }^{2}$ Kwokshing Chan, ${ }^{1}$ Lars Erwig, ${ }^{3}$
} Andrew Schrepf, ${ }^{4}$ Paula Dospinescu, ${ }^{2}$ Stuart Gray, ${ }_{1}^{5}$ Gordon Waiter, ${ }^{1}$ Jiabao He, ${ }^{1}$ and Neil Basu ${ }^{5}$

Objective. To investigate the potential structural and metabolic role of skeletal muscle in systemic lupus erythematosus (SLE)-related fatigue.

Methods. A case-control, multimodal magnetic resonance imaging (MRI) study was conducted. Cases were patients with inactive SLE who reported chronic fatigue. Controls were age- and sex-matched healthy members of the general population. Patients were clinically characterized and then underwent a 3T whole-body MRI scan. Resting and dynamic ${ }^{31} \mathrm{P} \mathrm{MRI}$ spectroscopy of the calf muscles was applied, from which phosphocreatine (PCr) recovery halftime, a marker of mitochondrial dysfunction, was computed. In addition, microstructural sequences (T1-weighted anatomic images, T2 mapping, and diffusion tensor imaging) were acquired. Descriptive statistics evaluated group differences and within-case physical fatigue correlations were explored.

Results. Of the 37 recruits (mean age 43.8 years, $89.2 \%$ female), cases $(n=19)$ reported higher levels of physical fatigue, pain, depression, and sleep disturbance compared to the control group $(P<0.0001)$. PCr was greater $(P=$ 0.045 ) among cases (mean \pm SD $33.0 \pm 9.0$ seconds) compared to controls (mean \pm SD $27.1 \pm 6.6$ seconds). No microstructural group differences were observed. Within cases, physical fatigue did not correlate with $\operatorname{PCr}(r=-0.28$, $P=0.25)$.

Conclusion. We report preliminary data demonstrating greater skeletal muscle mitochondrial dysfunction among fatigued patients with SLE compared to healthy controls.

\section{INTRODUCTION}

Patients with systemic lupus erythematosus (SLE) consider fatigue to be one of the most pervasive and disabling aspects of their disease. As many as $85 \%$ of patients report significant levels of fatigue (1), a prevalence greater than that observed in the general population and among patients with more common inflammatory rheumatic disorders (2). Moreover, the impact of fatigue permeates all aspects of life, as reflected by its strong associations with impaired quality of life (3) and work disability (4). Despite these significant consequences, little is understood about this symptom. The major challenge in clinical practice is to deliver therapeutic options to those patients whose disease is otherwise in remission and for whom no other reversible causes are apparent (5).

\footnotetext{
Supported by Lupus UK.

${ }^{1}$ Sai Man Cheung, MSc, Karen Keenan, PhD, Nicholas Senn, MSc, Kwokshing Chan, MSc, Gordon Waiter, PhD, Jiabao He, PhD: University of Aberdeen, Aberdeen, UK; 'ayle Hutcheon, BSc, Paula Dospinescu, MD: NHS Grampian, Aberdeen, UK; ${ }^{3}$ Lars Erwig, MD, PhD: University of Aberdeen, Aberdeen, and GlaxoSmithKline, Stevenage, UK; ${ }^{4}$ Andrew Schrepf, PhD: University of Michigan, Ann Arbor; ${ }^{5}$ Stuart Gray, PhD, Neil Basu, MD, PhD: University of Glasgow, Glasgow, UK.
}

Patients describe multiple dimensions of fatigue, and therefore its etiology is likely to be complex. The predominance of both physical and mental fatigue (6) alludes to a mixture of peripheral and central mechanisms. In terms of investigating the former, skeletal muscle dysfunction has previously been associated with SLErelated fatigue (7), although no studies have investigated whether this observation is underpinned by pathologic abnormalities within the muscles themselves.

Developments in magnetic resonance imaging (MRI) technology offer a noninvasive opportunity to comprehensively quantify skeletal muscle pathology at both metabolic and structural levels. For example, ${ }^{31} \mathrm{P}$ MRI spectroscopy (MRS) allows for the direct measurement of altered metabolic activity, such as levels of phosphocreatine (PCr), in vivo during physical activity, and MRS has previously signaled dysfunction in the muscles of chronic fatigue

No potential conflicts of interest relevant to this article were reported.

Address correspondence to Neil Basu, MD, PhD, Institute of Infection, Immunology and Inflammation, University of Glasgow, Glasgow, G12 8TA, UK. E-mail: neil.basu@glasgow.ac.uk.

Submitted for publication May 31, 2018; accepted in revised form January 8, 2019. 


\section{SIGNIFICANCE \& INNOVATIONS}

- Systemic lupus erythematosus (SLE)-related fatigue does not appear to be implicated with abnormal skeletal muscle microstructure.

- Patients with SLE exhibit higher levels of skeletal muscle mitochondrial dysfunction.

syndrome (CFS) populations (8). In contrast to CFS, there is some histologic evidence that at least selected patients with SLE exhibit structural abnormalities in their skeletal muscle (9). Novel methods, such as diffusion tensor imaging (DTI), are sensitive to pathologic abnormalities associated with overall cell geometry and edema $(10,11)$. In addition to high-resolution MRI for the quantification of muscle volume, T2 mapping highlights edema, while Dixon MRI allows quantitative measurement of fat infiltration. To our knowledge, no study has yet to contemporaneously use these methodologic advances to investigate SLE. We aimed to investigate the differences between the metabolic and structural features of skeletal muscle among patients with SLE with idiopathic fatigue and healthy controls using multimodal MRI muscle imaging.

\section{PATIENTS AND METHODS}

A case-control study was conducted. Subjects were invited to undertake a multimodal MRI scan of their calf muscles alongside the collection of clinical data. The East Midlands-Leicester Research Ethics Committee (ref: 15/EM/0418) approved the study, and written informed consent was obtained from patients according to the Declaration of Helsinki.

Patients. Cases were patients with SLE, classified according to the 1997 American College of Rheumatology criteria (12), attending secondary care clinics in NHS Grampian. To be eligible, patients were required to report chronic (>3 months), clinically important fatigue (defined as a score of $>3$ on the Chalder Fatigue Scale [binary scoring]) (13), experience reduced muscle strength (item 6 of the Chalder Fatigue Scale), and have inactive SLE, defined as a British Isles Lupus Assessment Group 2004 score of $O$ (excluding the fatigue constitutional domain) (14). In addition, patients were excluded if they had any past history of clinically diagnosed myositis or alternative medical explanations for their fatigue (symptomatic cardiorespiratory disease, a history of cancer in the previous 5 years, unstable thyroid disease, moderateto-severe chronic kidney disease, moderate-to-severe anemia, a beta-blocker prescription, or fibromyalgia).

Controls, recruited by local advertising, were healthy (no relevant past medical history) subjects who did not report clinically important fatigue (Chalder Fatigue Scale $\leq 3$ ) or reduced muscle strength (item 6 of the Chalder Fatigue Scale). They were approximately matched to cases by age and sex. In order to off- set potential confounding due to deconditioning, controls were additionally required to be sedentary, defined as those having a desk job and undertaking $<3$ hours of physical activity per week (8). Any potential case or control with a contraindication to MRI (e.g., pacemaker in situ) was excluded.

Clinical characterization. Eligible cases underwent a clinical evaluation that included an assessment of disease damage (according to Systemic Lupus International Collaborating Clinics [SLICC] criteria) (15), previous organ involvement, and disease duration. Erythrocyte sedimentation rate, serum creatinine, and creatine kinase were measured in both cases and controls. All subjects completed a self-reported questionnaire that included the following validated measures and known confounders of fatigue: 1) the Chalder Fatigue Scale is one of the most commonly employed measures of fatigue and has been found to be both feasible and acceptable in SLE (16); of the 11 questions, 7 specifically examine physical fatigue and are scored on a Likert scale (range 0-21), with high scores indicating high levels of physical fatigue; 2) the Hospital Anxiety and Depression Scale is a validated 14-item tool for assessing anxiety and depression in patients with SLE and in the general population (17); this scale also employs a Likert-style scoring system (range 0-21 for each domain); 3) pain severity was measured using a 0-10 numerical rating scale; and 4) Jenkin's Sleep Scale is known to perform well in both nonclinical and clinical populations, succinctly quantifying key sleep dysfunction domains, i.e., difficulties in sleep onset and maintenance, early wakening, and nonrestorative sleep; the domain scores are totaled (range 0-20), with higher scores indicating greater sleep disturbance (18).

Finally, both cases and controls underwent the Siconolfi Step Test. This measure of aerobic fitness (a putative confounder) has been validated in patients with SLE (19). It involved patients stepping up and down from a 10-inch bench for 3 minutes at a rate of 17 steps per minute (guided by a metronome). Heart rate was monitored and the protocol stopped if $65 \%$ of the predicted heart rate (220 minus age) was exceeded. If not reached, then a second stage (26 steps per minute) and a third stage (34 steps per minute) were performed, with 1-minute rest between stages. Maximal oxygen uptake was then estimated using the formulas described by Siconolfi et al (20).

MRI acquisition. Images were acquired on a 3T wholebody MRI scanner (Achieva TX, Philips Healthcare) using the body coil for transmission and an 8-channel knee coil as the receiver. In 1 patient with SLE and 2 healthy controls, the diameter of the lower leg was too large for the knee coil, and for these participants a 2-channel flex-M receiver coil was used. The imaging volume was centered at the thickest part of the right calf, with the subject in supine position. 
T1-weighted anatomic images were acquired using a standard sequence with repetition time (TR) of 2,700 msec, echo time (TE) of $55 \mathrm{msec}$, field of view (FOV) of $160 \times 160$ $\mathrm{mm}^{2}$, matrix size of $160 \times 160$, and 48 slices of $1.5-\mathrm{mm}$ thickness. T2 mapping was performed using a gradient and spin-echo sequence with TR of $3,137 \mathrm{msec}$, and with 12 equally spaced echoes from TE of $10-120$ msec $(21,22)$. DTI was acquired using a single-shot pulsed gradient spin-echo sequence with TR/TE of 2,000/53 msec $(11,23), 32$ diffusion directions, diffusion weighting of 400 seconds $/ \mathrm{mm}^{2}$, and 2 averages (24). Fat mapping was performed using a multislice multi-echo spoiled gradient-echo sequence, with TR of 20 msec, 16 equally spaced echoes from TE of 1.14 to 18.24 msec, and $3^{\circ}$ flip angle $(25,26)$. For T2 mapping, DTI, and fat mapping, the imaging volume was set to FOV of $192 \times 192$ $\mathrm{mm}^{2}$, and 12 transverse slices of $6-\mathrm{mm}$ thickness. The matrix size was set to $128 \times 128$ for T2 and fat mapping, and $64 \times 64$ for DTI, to ensure adequate signal-to-noise ratio (24).

${ }^{31} \mathrm{P}-\mathrm{MRS}$ scans were acquired from a $14-\mathrm{cm}$ diameter ${ }^{31} \mathrm{P}$ coil positioned underneath the thickest part of the calf, using a 1-D image-selected in vivo spectroscopy sequence with the detection slab covering the posterior portion of the calf (27). Dynamic spectra were acquired with TR of 5 seconds and 108 dynamics (28), while the subject concurrently performed a plantar flexion exercise protocol at $20 \%$ maximal voluntary contraction in synchrony to an audio metronome prompt at 35 beats per minute. Isometric maximal voluntary contraction of the right calf with $90^{\circ}$ plantar ankle flexion for each subject was measured (KinCom $500 \mathrm{H}$ dynamometer). Measurements at 2-minute intervals were performed until the difference between the last 2 measures was $>5 \%$ of their average; normally 3-4 repetitions were performed. The highest of the last
2 measures was taken as the maximal voluntary contraction (29). The exercise paradigm contained a 2-minute baseline followed by 2 8-minute cycles, where each cycle was composed of 3 minutes of exercise before a 5-minute recovery period.

Image analysis. The ${ }^{31} \mathrm{P}$ spectra were processed in jMRUI software, version 3.0 (30), and PCr halftime was computed from the PCr time course in the postexercise recovery period as an indicator of muscle energetics (31). DTI analysis was performed in FSL software FMRIB, to derive metrics maps of mean diffusion (MD), radial diffusivity (RD), and fractional anisotropy (FA) (23) as indicators of muscle integrity (24). In 5 subjects, images affected by motion artefact, resulting in failure of the motion correction algorithm, were identified and removed before the calculation of diffusion metrics. T2 maps were computed using in-house software in MATLAB (MathWorks), following standard procedures (21). Fat fraction maps, as the ratio between fat and the sum of fat and water images, were computed using the ISMRM Fat-Water Toolbox in MATLAB (32). Fat and water were separated using a multistep fitting approach (33), incorporating a multifrequency fat-spectrum model $(34,35)$. To avoid confounding factors, patients with nonadherence to the exercise protocol were excluded from the PCr halftime analysis; patients using the flex-M coil or showing severe image artefact were excluded from corresponding image analysis (Table 1).

Regions of interest were manually drawn by a single operator in MRIcron on the central 10 slices of the image acquired at a TE of $10 \mathrm{msec}$ from T2 mapping, to delineate soleus and exclude subcutaneous fat or blood vessels. The binary masks were subsequently applied on maps of $T 2, M D, F A, R D$, and fat

Table 1. Magnetic resonance imaging results*

\begin{tabular}{|c|c|c|c|c|}
\hline Result & SLE & Healthy controls & t-test scoret & P \\
\hline \multicolumn{5}{|l|}{ Metabolism‡ } \\
\hline PCr halftime, seconds & $33.0 \pm 9.0$ & $27.1 \pm 6.6$ & 2.087 & $0.045 \S$ \\
\hline End-exercise $\mathrm{pH}$ & $7.00 \pm 0.01$ & $7.01 \pm 0.01$ & 0.704 & 0.488 \\
\hline Muscle integrity $\mathbf{q}$ & & & & \\
\hline $\mathrm{MD}\left(\times 10^{-3} \mathrm{~mm}^{2}\right.$ seconds $\left.{ }^{-1}\right)$ & $1.57 \pm 0.07$ & $1.54 \pm 0.12$ & 0.850 & 0.401 \\
\hline $\mathrm{RD}\left(\times 10^{-3} \mathrm{~mm}^{2}\right.$ seconds $\left.^{-1}\right)$ & $1.39 \pm 0.07$ & $1.38 \pm 0.11$ & 0.597 & 0.554 \\
\hline FA & $0.21 \pm 0.02$ & $0.21 \pm 0.02$ & 1.212 & 0.234 \\
\hline \multicolumn{5}{|l|}{ Muscle condition\# } \\
\hline T2, msec & $33.2 \pm 1.5$ & $32.6 \pm 1.1$ & 1.355 & 0.185 \\
\hline Fat infiltration** & & & & \\
\hline Fat fraction, \% & $3.69 \pm 1.27$ & $3.90 \pm 1.81$ & -0.381 & 0.706 \\
\hline Size, $\mathrm{CSA} \mathrm{cm}^{2}$ & $21.8 \pm 3.7$ & $22.6 \pm 5.4$ & -0.497 & 0.623 \\
\hline
\end{tabular}

* Values are the mean \pm SD unless indicated otherwise. SLE = systemic lupus erythematosus; $\mathrm{PCr}=$ phosphocreatine; $\mathrm{MD}=$ mean diffusivity; $\mathrm{RD}=$ radial diffusivity; $\mathrm{FA}$ = fractional anisotropy; $\mathrm{CSA}=$ cross-sectional area.

† Independent sample.

¥ One case and 1 control not analyzed due to exercise nonadherence. Two cases and 1 control not analyzed due to an artifact in the recovery curve.

$\S$ Statistically significant.

9 One control not analyzed due to image artifact.

\# Two cases and 2 controls not analyzed due to image artifacts.

** One case and 2 controls not analyzed due to flex-M coil use. One case not analyzed due to image artifact. 
Table 2. Baseline characteristics*

\begin{tabular}{lccc}
\multicolumn{1}{c}{ Characteristic } & $\begin{array}{c}\text { Cases } \\
(n=19)\end{array}$ & $\begin{array}{c}\text { Controls } \\
(n=18)\end{array}$ & $P \dagger$ \\
\hline $\begin{array}{l}\text { Demographics } \\
\text { Age, years }\end{array}$ & $44.8 \pm 14.43$ & $42.8 \pm 13.6$ & 0.67 \\
$\quad$ Female, no. & 17 & 16 & $0.95 \ddagger$ \\
Symptoms & $14.7 \pm 3.6$ & $6.9 \pm 0.6$ & $<0.0001$ \\
Physical fatigue (CFS) & $9.3 \pm 4.2$ & $4.3 \pm 2.4$ & 0.0001 \\
Anxiety (HADS) & $6.7 \pm 3.4$ & $1.6 \pm 1.7$ & $<0.0001$ \\
Depression (HADS) & $3.5 \pm 2.3$ & $0.3 \pm 0.8$ & $<0.0001$ \\
Pain (NRS; range 0-10) & $12.7 \pm 5.3$ & $4.8 \pm 5.3$ & $<0.0001$ \\
Sleep disturbance (ISS) & & & \\
Physiologic measures & $28.0 \pm 4.4$ & $28.4 \pm 6.0$ & 0.78 \\
Vor max (ml/kg/minute) & $18.7 \pm 14.2$ & $13.6 \pm 10.4$ & 0.28 \\
ESR (mm/hour) & $132.6 \pm 11.2$ & $131.1 \pm 6.9$ & 0.67 \\
Hemoglobin (gram/liter) & $69.7 \pm 24.0$ & $64.2 \pm 13.7$ & 0.39 \\
Creatinine ( $\mu$ moles/liter) & $89.4 \pm 34.2$ & $113.8 \pm 71.3$ & 0.20 \\
Creatinine kinase (U/liter) & & & \\
\hline
\end{tabular}

* Values are the mean \pm SD unless indicated otherwise. CFS $=$ Chalder Fatigue Scale (physical domain); HADS = Hospital Anxiety and Depression Scale; NRS = numeric rating scale; JSS = Jenkin's Sleep Scale; ESR = erythrocyte sedimentation rate. t Derived from $t$-tests unless indicated otherwise.

¥ Derived from chi-square test.

$\S$ Derived from Siconolfi Step Test.

fraction to generate the average value. Muscle volumes of soleus were also quantified as the cross-sectional area on the central slice of the T1-weighted anatomic image (36). Eighteen subjects per group sufficiently afforded $>80 \%$ power to detect an effect size of 0.85 , with a measurement error of $30 \%$ at an alpha of 0.05 (as measured by PCr recovery halftime).

Statistical analysis. Clinical parameters were expressed using simple descriptive statistics with case-control comparisons made using chi-square tests for categorical variables and $t$-tests for continuous variables. To investigate the role of skeletal muscle energetics in SLE, the case-control comparison of PCr halftime was performed using a $t$-test. To examine the role of muscle microstructure integrity and muscle volume in SLE, the case-control comparison of MD, FA, RD, T2, and fat fraction, as well as the cross-sectional area, was performed using t-tests. Within-case Pearson correlations were conducted using STATA software, version 12.1, to further investigate any identified group differences. Due to the small sample size, these analyses were considered exploratory.

\section{RESULTS}

Among the 37 recruited subjects (mean age 43.8 years, $89.2 \%$ female), cases ( $n=19$ ) reported significantly higher levels of physical fatigue, pain, depression, anxiety, and sleep disturbance compared to the control group, although the groups were comparable in terms of demographic and physiologic parameters (Table 2).

Overall, cases had mild SLE; only 1 patient had a history of renal involvement, and the mean \pm SD SLICC score was 0.11 \pm 0.3 . The majority of patients experienced musculoskeletal
( $n=17$ ) and/or cutaneous ( $n=12$ ) involvement. The most commonly prescribed immunosuppressant treatment was hydroxychloroquine $(n=15)$, followed by methotrexate $(n=8)$. Other SLE-specific treatment at the time of the study included azathioprine ( $n=2)$, mycophenolate mofetil $(n=3)$, and rituximab $(n=3)$. Only 3 patients were receiving long-term prednisolone (5-8 mg/day).

MRI analysis. In assessment of calf muscle metabolic function, there was a difference $(P=0.045)$ in the PCr halftime recovery between patients with SLE (mean \pm SD $33.0 \pm 9.0 \mathrm{sec}$ onds) and healthy controls (mean \pm SD $27.1 \pm 6.6$ seconds). There were no significant differences in MD, RD, or FA from DTI between patients with SLE and controls (Table 1). Additionally, there were no significant differences in T2 $(P=0.185)$ or fat fraction $(P=0.706)$ between patients with SLE (mean \pm SD T2 $33.2 \pm$ 1.5 msec; fat fraction $3.69 \pm 1.27 \%$ ) and controls (mean \pm SD T2 $32.6 \pm 1.1 \mathrm{msec}$; fat fraction $3.90 \pm 1.81 \%)$ or in muscle crosssectional areas $(P=0.623)$ (mean \pm SD SLE $21.8 \pm 3.7 \mathrm{~cm}^{2}$; controls $\left.22.6 \pm 5.4 \mathrm{~cm}^{2}\right)$. The MRI data from a healthy control are shown in Figure 1. There were no significant correlations identified between PCr halftime and levels of physical fatigue $(r=-0.28$ [95\% confidence interval $(95 \% \mathrm{Cl})-0.60,0.13], P=0.25)$, or mental fatigue $(r=0.2[95 \% \mathrm{Cl}-0.2,-0.54], P=0.41)$.

\section{DISCUSSION}

To the best of our knowledge, this is the first study of a rheumatic disease to investigate the relationship between skeletal muscle and fatigue employing multimodal MRI. Among fatigued patients with SLE, calf muscle PCr recovery halftime was significantly prolonged compared to nonfatigued healthy controls. 
A

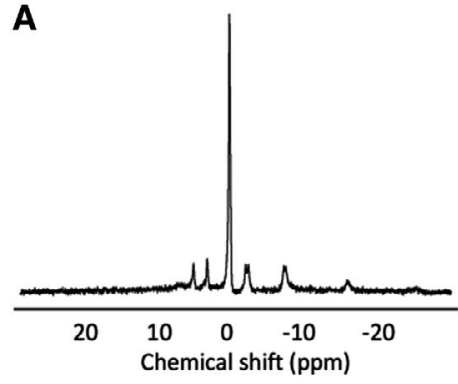

D

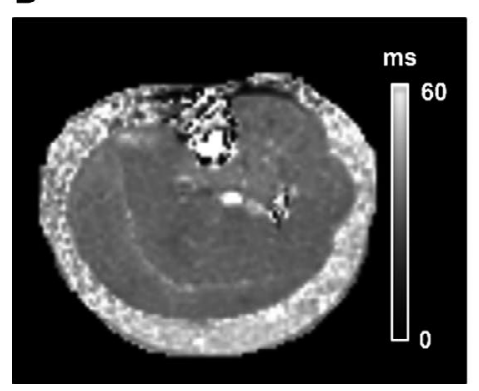

B

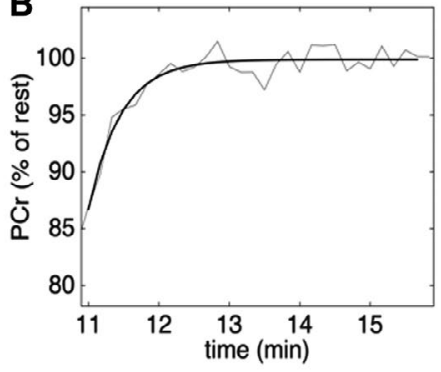

E

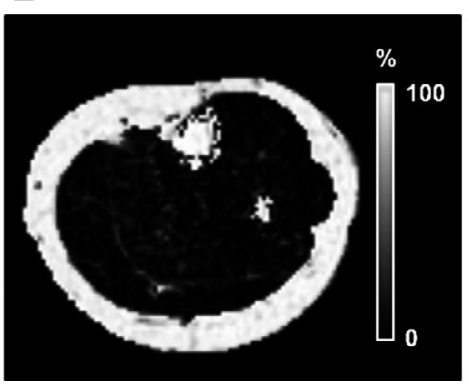

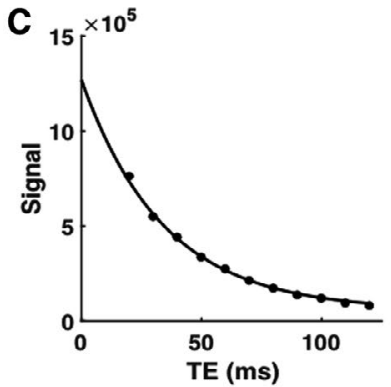

$\mathbf{F}$

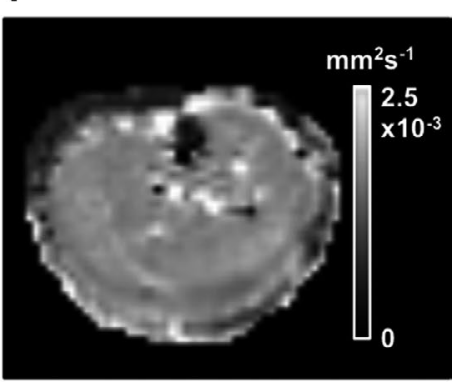

Figure 1. Magnetic resonance imaging data from a healthy control. A, Baseline ${ }^{31} \mathrm{P}$ spectrum; B, Dynamics of phosphocreatine (PCr) during the recovery period; C, Transverse relaxation from a single voxel within soleus muscle, shown together with fitted curve; D, Calculated transverse relaxation time map; $\mathbf{E}$, Fat fraction map; and F, mean diffusivity map. TE = echo time; $m s$ = milliseconds.

These differences do not appear to Obe related to physical fatigue. Further, no differences in skeletal muscle microstructure were observed between cases and controls. Taken together, skeletal muscle does not appear to serve as a major factor in SLE-related fatigue.

PCr recovery halftime reflects the muscle oxidative capacity and is used as a marker of muscle mitochondrial function (37). In SLE there is accumulating evidence to support the presence of mitochondrial abnormalities in peripheral blood cells. For example, Gergely et al observed hyperpolarized mitochondria in T cells that resulted in greater ATP depletion, oxidative stress, and ultimately cell death (38). We now provide supporting data that mitochondrial dysfunction might also exist within the skeletal muscle of patients with SLE. The same marker has previously been related to fatigue in SLE (39), although our exploratory analysis suggests that pathways other than skeletal muscle mitochondrial dysfunction may be involved in the generation of this symptom.

Microstructural MRI of skeletal muscles has been applied in only a few clinical populations and, to our knowledge, never in the investigation of fatigue. DTI has evidenced changes of muscle integrity in athletes following marathon runs, where standard sequences have failed to detect macroscopic differences (40). Furthermore, this method can distinguish disease activity in inflammatory muscle diseases with greater sensitivity than standard imaging (41). Among neuromuscular conditions, where existing clinical tests are inadequate to assess disease progression, the quantification of structural parameters such as muscle volumes and fat infiltration are providing superior biomarkers for clinical trials and practice (42). Such studies are similar in size to the present investigation, and so the absence of differences between our cases and controls in any of the sensitive microstructural metrics contradicts the hypothesis that physical fatigue is related to structural abnormalities in SLE skeletal muscle.

If not skeletal muscles, what then are the main explanations of physical fatigue among patients with SLE? A recent study of fatigue in another multisystem autoimmune disorder (antineutrophil cytoplasmic antibody-associated vasculitis) failed to detect a significant relationship between physical fatigue and skeletal muscle mass (measured using dual-energy $\mathrm{x}$-ray absorptiometry) or function. Compared to healthy controls, fatigued cases evidenced reduced voluntary activation of skeletal muscle and reduced maximal voluntary contraction of skeletal muscle, and they had higher levels of perceived exertion, a finding that significantly correlated with physical fatigue (43). Together, these observations pointed toward centrally rather than peripherally driven mechanisms.

The novel application of cutting-edge MRI methods combined with a comprehensive approach to phenotyping are strengths of this study, but a number of limitations must also be considered. First, the highly selective eligibility criteria (purposely planned to enhance homogeneity by excluding known fatigue mechanisms) has resulted in a sample with generally mild disease. The results are therefore not generalizable to the wider disease spectrum. For example, patients 
with a history of myositis (prevalent in $4-16 \%$ of SLE cases [44]) were excluded. Data from this study cannot be used to inform the usefulness of these methodologies in the evaluation of such manifestations (a distinct research question). Second, we recognize that patients with SLE without fatigue would have served as a more precise control group. That said, given the pervasiveness of fatigue in this disease, recruiting such patients would have been logistically challenging. Regardless, the absence of differences even with a healthy control group (as observed with almost all of the MRI metrics) indicates that these methodologies are unlikely to identify a clinically relevant fatigue-specific signal. Uncertainty also exists regarding the clinical relevance of the statistically significant $\mathrm{PCr}$ measure, since the 6-second difference in recovery halftime is lower in magnitude compared to other ${ }^{31} \mathrm{P}$ studies (for example, mean \pm SD $18.7 \pm 0.9$ seconds in healthy controls versus $27.3 \pm$ 3.5 seconds in patients with diabetes mellitus [45], or mean \pm SD $35.0 \pm 3.0$ seconds in healthy controls versus $45.0 \pm$ 4.0 seconds in patients with chronic obstructive pulmonary disease [46]). Third, although the sample size is equivalent to other MRI muscle studies, which have detected significant changes in other populations, we cannot be certain that larger sample sizes will not identify a significant effect. In particular, fully powered analyses of within-case correlational analysis might uncover relationships between PCr and SLE fatigue. We suspect, however, that in the absence of even a trend, any associations are unlikely to be major contributors to our understanding of physical fatigue.

This study provides evidence of feasibility for the use of multimodal MRI muscle assessment in patients with SLE. From this data, the investigation of physical fatigue would seem to be better served by examining alternatives to skeletal muscle-based pathways. Learning from other chronic diseases, the investigation of central mechanisms using advanced MRI brain techniques appears to offer greater potential (47). Such approaches have been limited in SLE and should be encouraged in an effort to better understand this considerable patient challenge.

\section{ACKNOWLEDGMENT}

The authors thank the patient community who participated in this research effort.

\section{AUTHOR CONTRIBUTIONS}

All authors were involved in drafting the article or revising it critically for important intellectual content, and all authors approved the final version to be submitted for publication. Dr. Basu had full access to all of the data in the study and takes responsibility for the integrity of the data and the accuracy of the data analysis.

Study conception and design. Erwig, Gray, Waiter, He, Basu.

Acquisition of data. Keenan, Hutcheon, Dospinescu, Basu.

Analysis and interpretation of data. Cheung, Senn, Chan, Schrepf, Waiter, He, Basu.

\section{REFERENCES}

1. Zonana-Nacach A, Roseman JM, McGwin G Jr, Friedman AW, Baethge BA, Reveille JD, et al. Systemic lupus erythematosus in three ethnic groups. VI: factors associated with fatigue within 5 years of criteria diagnosis. LUMINA Study Group. LUpus in MInority populations: NAture vs Nurture. Lupus 2000;9:101-9.

2. Overman CL, Kool MB, Da Silva JA, Geenen R. The prevalence of severe fatigue in rheumatic diseases: an international study. Clin Rheumatol 2016;35:409-15.

3. Wang B, Gladman DD, Urowitz MB. Fatigue in lupus is not correlated with disease activity. J Rheumatol 1998;25:892-5.

4. Baker K, Pope J, Silverman E, Cooper G, Fortin P, Zummer M, et al. Work disability in systemic lupus erythematosus is prevalent and predicted by socio-demographic and disease related factors: results from a multi-ethnic cohort. J Rheumatol 2009;36:2559-60.

5. Cleanthous S, Tyagi M, Isenberg DA, Newman SP. What do we know about self-reported fatigue in systemic lupus erythematosus? Lupus 2012;21:465-76.

6. Da Costa D, Dritsa M, Bernatsky S, Pineau C, Menard HA, Dasgupta $\mathrm{K}$, et al. Dimensions of fatigue in systemic lupus erythematosus: relationship to disease status and behavioral and psychosocial factors. J Rheumatol 2006;33:1282-8.

7. Balsamo S, da Mota LM, de Carvalho JF, Nascimento DD, Tibana RA, de Santana FS, et al. Low dynamic muscle strength and its associations with fatigue, functional performance, and quality of life in premenopausal patients with systemic lupus erythematosus and low disease activity: a case-control study. BMC Musculoskelet Disord 2013;14:263.

8. Jones DE, Hollingsworth KG, Taylor R, Blamire AM, Newton JL. Abnormalities in $\mathrm{pH}$ handling by peripheral muscle and potential regulation by the autonomic nervous system in chronic fatigue syndrome. J Intern Med 2010;267:394-401.

9. Lim KL, Abdulwahab R, Lowe J, Powell RJ. Muscle biopsy abnormalities in systemic lupus-erythematosus: correlation with clinical and laboratory parameters. Ann Rheum Dis 1994;53:178-82.

10. Ai T, Yu K, Gao L, Zhang P, Goerner F, Runge VM, et al. Diffusion tensor imaging in evaluation of thigh muscles in patients with polymyositis and dermatomyositis. Br J Radiol 2014;87:20140261.

11. Galban CJ, Maderwald S, Stock F, Ladd ME. Age-related changes in skeletal muscle as detected by diffusion tensor magnetic resonance imaging. J Gerontol A Biol Sci Med Sci 2007;62:453-8.

12. Hochberg MC, for the Diagnostic and Therapeutic Criteria Committee of the American College of Rheumatology. Updating the American College of Rheumatology revised criteria for the classification of systemic lupus erythematosus [letter]. Arthritis Rheum 1997;40:1725

13. Chalder T, Berelowitz G, Pawlikowska T, Watts L, Wessely S, Wright $D$, et al. Development of a fatigue scale. J Psychosom Res 1993;37:147-53.

14. Isenberg DA, Rahman A, Allen E, Farewell V, Akil M, Bruce IN, et al. BILAG 2004: development and initial validation of an updated version of the British Isles Lupus Assessment Group's disease activity index for patients with systemic lupus erythematosus. Rheumatology (Oxford) 2005;44:902-6.

15. Gladman D, Ginzler E, Goldsmith C, Fortin P, Liang M, Urowitz M, et al. The development and initial validation of the Systemic Lupus International Collaborating Clinics/American College of Rheumatology damage index for systemic lupus erythematosus. Arthritis Rheum 1996;39:363-9.

16. Goligher EC, Pouchot J, Brant R, Kherani RB, Avina-Zubieta JA, Lacaille D, et al. Minimal clinically important difference for 7 measures of fatigue in patients with systemic lupus erythematosus. $J$ Rheumatol 2008;35:635-42. 
17. Holloway L, Humphrey L, Heron L, Pilling C, Kitchen H, Hojbjerre $L$, et al. Patient-reported outcome measures for systemic lupus erythematosus clinical trials: a review of content validity, face validity and psychometric performance. Health Qual Life Outcomes 2014;12:116

18. Jenkins CD, Stanton BA, Niemcryk SJ, Rose RM. A scale for the estimation of sleep problems in clinical research. J Clin Epidemiol 1988;41:313-21.

19. Marcora SM, Casanova F, Fortes MB, Maddison PJ. Validity and reliability of the Siconolfi Step Test for assessment of physical fitness in patients with systemic lupus erythematosus. Arthritis Rheum 2007;57:1007-11.

20. Siconolfi SF, Garber CE, Lasater TM, Carleton RA. A simple, valid step test for estimating maximal oxygen uptake in epidemiologic studies. Am J Epidemiol 1985;121:382-90.

21. Arpan I, Forbes SC, Lott DJ, Senesac CR, Daniels MJ, Triplett WT, et al. $T_{2}$ mapping provides multiple approaches for the characterization of muscle involvement in neuromuscular diseases: a crosssectional study of lower leg muscles in 5-15-year-old boys with Duchenne muscular dystrophy. NMR Biomed 2013;26:320-8.

22. Schwenzer NF, Martirosian P, Machann J, Schraml C, Steidle G, Claussen $C D$, et al. Aging effects on human calf muscle properties assessed by MRI at 3 Tesla. J Magn Reson Imaging 2009;29:134654.

23. Scheel M, von Roth P, Winkler T, Arampatzis A, Prokscha T, Hamm $\mathrm{B}$, et al. Fiber type characterization in skeletal muscle by diffusion tensor imaging. NMR Biomed 2013;26:1220-4.

24. Sinha U, Csapo R, Malis V, Xue YJ, Sinha S. Age-related differences in diffusion tensor indices and fiber architecture in the medial and lateral gastrocnemius. J Magn Reson Imaging 2015;41:941-53.

25. Liu CY, McKenzie CA, Yu H, Brittain JH, Reeder SB. Fat quantification with IDEAL gradient echo imaging: correction of bias from T-1 and noise. Magn Reson Med 2007;58:354-64.

26. Kuhn JP, Jahn C, Hernando D, Siegmund W, Hadlich S, Mayerle J, et al. T1 bias in chemical shift-encoded liver fat-fraction: role of the flip angle. J Magn Reson Imaging 2014;40:875-83.

27. Sprott H, Rzanny R, Reichenbach JR, Kaiser WA, Hein G, Stein G. $\mathrm{P}-31$ magnetic resonance spectroscopy in fibromyalgic muscle. Rheumatology (Oxford) 2000;39:1121-5.

28. Wu JS, Buettner C, Smithline H, Ngo LH, Greenman RL. Evaluation of skeletal muscle during calf exercise by 31 -phosphorus magnetic resonance spectroscopy in patients on statin medications. Muscle Nerve 2011;43:76-81.

29. Bentley R, Gray SR, Schwarzbauer C, Dawson D, Frenneaux M, He J. Dietary nitrate reduces skeletal muscle oxygenation response to physical exercise: a quantitative muscle functional MRI study. Physiol Rep 2014;2:e12089.

30. Naressi A, Couturier C, Castang I, de Beer R, Graveron-Demilly D. Java-based graphical user interface for MRUI, a software package for quantitation of in vivo/medical magnetic resonance spectroscopy signals. Comput Biol Med. 2001;31:269-86.

31. Haseler LJ, Hogan MC, Richardson RS. Skeletal muscle phosphocreatine recovery in exercise-trained humans is dependent on O-2 availability. J Appl Physiol 1999;86:2013-8.
32. Hu HH, Bornert P, Hernando D, Kellman P, Ma JF, Reeder S, et al. ISMRM workshop on fat-water separation: insights, applications and progress in MRI. Magn Reson Med 2012;68:378-88.

33. Zhong XD, Nickel MD, Kannengiesser SA, Dale BM, Kiefer B, Bashir MR. Liver fat quantification using a multi-step adaptive fitting approach with multi-echo GRE imaging. Magn Reson Med 2014;72:1353-65.

34. Ren JM, Dimitrov I, Sherry AD, Malloy CR. Composition of adipose tissue and marrow fat in humans by $1 \mathrm{H}$ NMR at 7 Tesla. J Lipid Res 2008;49:2055-62.

35. Yu HZ, Shimakawa A, McKenzie CA, Brodsky E, Brittain JH, Reeder SB. Multiecho water-fat separation and simultaneous $\mathrm{R}-2^{*}$ estimation with multifrequency fat spectrum modeling. Magn Reson Med 2008;60:1122-34.

36. Wiewiorski M, Dopke K, Steiger C, Valderrabano V. Muscular atrophy of the lower leg in unilateral post traumatic osteoarthritis of the ankle joint. Int Orthop 2012;36:2079-85.

37. Hollingsworth KG, Newton JL, Taylor R, McDonald C, Palmer JM, Blamire AM, et al. Pilot study of peripheral muscle function in primary biliary cirrhosis: potential implications for fatigue pathogenesis. Clin Gastroenterol Hepatol 2008;6:1041-8.

38. Gergely P Jr, Grossman C, Niland B, Puskas F, Neupane H, Allam F, et al. Mitochondrial hyperpolarization and ATP depletion in patients with systemic lupus erythematosus. Arthritis Rheum 2002;46:175-90.

39. Segal BM, Thomas W, Zhu X, Diebes A, McElvain G, Baechler E, et al. Oxidative stress and fatigue in systemic lupus erythematosus. Lupus 2012;21:984-92.

40. Froeling M, Oudeman J, Strijkers GJ, Maas M, Drost MR, Nicolay K, et al. Muscle changes detected with diffusion-tensor imaging after long-distance running. Radiology 2015;274:548-62.

41. Qi J, Olsen NJ, Price RR, Winston JA, Park JH. Diffusion-weighted imaging of inflammatory myopathies: polymyositis and dermatomyositis. J Magn Reson Imaging 2008;27:212-7.

42. Morrow JM, Sinclair CD, Fischmann A, Machado PM, Reilly MM, Yousry TA, et al. MRI biomarker assessment of neuromuscular disease progression: a prospective observational cohort study. Lancet Neurol 2016;15:65-77.

43. McClean A, Morgan MD, Basu N, Bosch JA, Nightingale P, Jones D, et al. Physical fatigue, fitness, and muscle function in patients with antineutrophil cytoplasmic antibody-associated vasculitis. Arthritis Care Res (Hoboken) 2016;68:1332-9.

44. Liang Y, Leng RX, Pan HF, Ye DQ. Associated variables of myositis in systemic lupus erythematosus: a cross-sectional study. Med Sc Monit 2017;23:2543-9.

45. Schrauwen-Hinderling VB, Kooi ME, Hesselink MK, Jeneson JA, Backes $\mathrm{WH}$, van Echteld CJ, et al. Impaired in vivo mitochondrial function but similar intramyocellular lipid content in patients with type 2 diabetes mellitus and BMI-matched control subjects. Diabetologia 2007;50:113-20.

46. McKeough ZJ, Alison JA, Bye PT, Trenell MI, Sachinwalla T, Thompson $\mathrm{CH}$, et al. Exercise capacity and quadriceps muscle metabolism following training in subjects with COPD. Respir Med 2006;100:1817-25.

47. DeLuca J, Genova HM, Capili EJ, Wylie GR. Functional neuroimaging of fatigue. Phys Med Rehabil Clin North Am 2009;20:325-37. 